\title{
Sensitivity of tracer transport to model resolution, prescribed meteorology and tracer lifetime in the general circulation model ECHAM5
}

\author{
A. M. Aghedo ${ }^{1, *}$, S. Rast $^{1}$, and M. G. Schultz ${ }^{1, * *}$ \\ ${ }^{1}$ Max Planck Institute for Meteorology, Hamburg, Germany \\ * now at: Jet Propulsion Laboratory, California Institute of Technology, Pasadena, California, USA \\ *** now at: ICG-2, Research centre, Jülich, Germany
}

Received: 13 November 2007 - Published in Atmos. Chem. Phys. Discuss.: 8 January 2008

Revised: 23 December 2009 - Accepted: 26 March 2010 - Published: 12 April 2010

\begin{abstract}
Atmospheric transport of traces gases and aerosols plays an important role in the distribution of air pollutants and radiatively active compounds. For model simulations of chemistry-climate interactions it is important to know how the transport of tracers depends on the geographical resolution of the general circulation model. However, this aspect has been scarcely investigated until now. Here, we analyse tracer transport in the ECHAM5 general circulation model using 6 independent idealized tracers with constant lifetimes, which are released in two different altitudes at the surface and in the stratosphere, respectively. Model resolutions from T21L19 to T106L31 were tested by performing multi-annual simulations with prescribed sea surface temperatures and sea ice fields of the 1990s. The impacts of the tracer lifetime were investigated by varying the globally uniform exponential decay time between 0.5 and 50 months. We also tested the influence of using prescribed meteorological fields (ERA40) instead of climatological sea surface temperature and sea ice fields. Meridional transport of surface tracers decreases in the coarse resolution model due to enhanced vertical mixing, with the exception of the advection into the tropical region, which shows an inconsistent trend between the resolutions. Whereas, the meridional transport of tracers released in the stratosphere was enhanced with higher model resolutions, except in the transport from tropical stratosphere to the Southern Hemisphere, which exhibits an increase trend with increasing model resolution. The idealized tracers exhibit a seasonal cycle, which is modulated by the tracer lifetime. In comparison to the run with prescribed sea surface temperature and sea ice fields, the simulation with prescribed
\end{abstract}

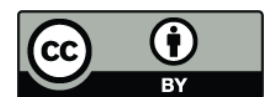

Correspondence to: A. M. Aghedo (adetutu.m.aghedo@jpl.nasa.gov) meteorological fields did not exhibit significant change in the meridional transport, except in the exchange of stratospheric tracers between both hemispheres, where it causes about $100 \%$ increase. The import of the surface tracers into the stratosphere is increased by up to a factor of 2.5 , and the export from the stratosphere into the troposphere was increased by up to $60 \%$ when prescribed meteorological fields is used. The ERA40 simulation also showed larger interannual variability (up to $24 \%$ compared to $12 \%$ in the standard simulations). Using our surface tracers released in either the northern or Southern Hemisphere, respectively, we calculate inter-hemispheric transport times between 11 and 17 months, consistent with values reported in the literature. While this study cannot be used to relate differences in model results to specific changes in transport processes, it nevertheless provides some insight into the characteristics of tracer transport in the widely used ECHAM5 general circulation model.

\section{Introduction}

Transport plays a crucial role in determining the distribution of gas-phase and particulate trace constituents in the atmosphere. Numerical models are an essential tool for simulating atmospheric transport and distribution of trace species. However, the ability of a model to simulate the observed distributions of these trace species is largely dependent on its capability to reproduce the transport and mixing of the real atmosphere. Gurney et al. $(2002,2003)$ show that differences in model transport are a significant source of uncertainty. Hall et al. (1999) concluded that transport inaccuracies significantly affect the simulation of important long-lived trace species in the lower stratosphere. Model

Published by Copernicus Publications on behalf of the European Geosciences Union. 
resolution also plays an important role. Genthon and Armengaud (1995) suggested that model spatial resolution could be an important factor in the simulation of the distribution of ${ }^{222} \mathrm{Rn}$, while Austin et al. (1997) and Wild and Prather (2006) demonstrated the influence of model resolution on the simulation of ozone distribution in the stratosphere and the troposphere, respectively.

Idealized tracers have previously been employed to investigate specific features of atmospheric transport, for example Gray (2003) used passive tracers to study the influence of convection on the stratosphere - troposphere exchange of air. Also by comparing the results from different model resolutions, idealized tracers may explain some of the discrepancies observed in the distribution or seasonality of atmospheric trace species in different models (e.g. Genthon and Armengaud, 1995; Jacob et al., 1997; Denning et al., 1999; Stevenson et al., 2006).

In a special issue of the Journal of Climate, titled "Climate Models at the Max Planck Institute for Meteorology (MPIM)" (Vol. 19, Issue 16, August 2006), the influence of model resolution on simulated climate in the ECHAM5 (Roeckner et al., 2003) general circulation model (GCM) was presented by Roeckner et al. (2006). This study extends the analysis of the ECHAM5 model by testing the sensitivity of the transport of tracers emitted at 6 different locations in the model, in order to answer three major questions: (1) How sensitive is the transport of tracers to model resolution? (2) How does a change in prescribed meteorology and tracer lifetime affect tracer transport? (3) What are the characteristic time scales for inter-hemispheric transport?

A brief description of the ECHAM5 model and the details of the model set-up are given in Sect. 2. The results are presented in Sect. 3 through 5. These include the analysis of the global characteristics of tracer transport in Sect. 3, the discussion of the transport from the source regions into various receptor regions in Sect. 4, and the calculation of the interhemispheric transport time in Sect. 5. The summary of our findings is presented in Sect. 6 .

\section{Model description}

\subsection{The ECHAM5 general circulation model}

The atmospheric general circulation model ECHAM5 is the fifth-generation climate model developed at the Max Planck Institute for Meteorology, evolving originally from the model of the European Centre for Medium-range Weather Forecast (ECMWF) (Simmons et al., 1989). The dynamical core of ECHAM5 solves prognostic equations for vorticity, divergence, logarithm of surface pressure and temperature, which are expressed in the horizontal by spectral coefficients. The model uses a semi-implicit leapfrog time integration scheme (Robert et al., 1972; Robert, 1981, 1982) and a special time filter (Asselin, 1972). The vertical axis uses a hy- brid sigma-pressure coordinate system and finite-difference scheme (Simmons and Burridge, 1981). The finite-difference scheme is implemented such that energy and angular momentum are conserved.

Advection of tracers is parameterized with the flux-form semi-Lagrangian transport scheme (Lin and Rood, 1996) on a Gaussian grid (Arakawa C-grid, Mesinger and Arakawa, 1976). The trace constituents are not subject to horizontal diffusion within the model, however surface emissions and other trace constituents undergo vertical diffusion, which are obtained from the bulk transfer relationships involving the difference of the respective model variable (wind components, potential temperature, humidity) between the surface and the lowest model level (about $30 \mathrm{~m}$ above ground), the wind velocity at that level, and the transfer coefficients. The transfer coefficients are obtained from Monin-Obukhov similarity theory (for details see Roeckner et al. (2003)). A mass flux scheme is employed for shallow, midlevel, and deep convection (Tiedtke, 1989) with modifications for deep convection according to Nordeng (1994). Subgrid-scale orography interacts with atmospheric flow in two ways in ECHAM5: orographic gravity waves transfer momentum from the earth to the atmosphere and they exert a drag when the air flow is blocked at low levels. The subgrid-scale orography is parameterised according to Lott and Miller (1997).

A detailed model description is given in Roeckner et al. (2003). The influence of model resolution on simulated climate in the ECHAM5 general circulation model (GCM) was presented by Roeckner et al. (2006), with the discussions on the systematic errors and convergence properties of the simulated climate in the model. They found little evidence for convergence to a more realistic climate state at horizontal resolutions greater than T42 in the L19 resolutions, and that the root-mean-square errors (RMSE) decreases monotonically with increasing horizontal resolution from T42 to T159 in the model version containing 31 levels (L31).

ECHAM5 contains a flexible software structure for definining atmospheric tracers. These tracers are then subjected to advection, convection and vertical diffusion. These transport processes are calculated separately using an operator splitting method. In ECHAM5, advective transport is done first, followed by vertical diffusion, chemical reactions or exponential decay, and in a last step convective transport. Each transport process is calculated from the knowledge of the tracer concentration at the preceding time step except the convection in which the tracer concentration updated by the previous processes is used. The resulting tendencies of each single process are added to the concentration of the previous time step.

ECHAM5 can be run as a coupled ocean-atmosphere model, or with forced boundaries from prescribed sea surface temperatures and sea ice cover fields. In addition, a Newtonian relaxation technique, also termed "nudging" (Hoke and Anthes, 1976; Jeuken et al., 1996) can be applied in order to simulate real weather episodes. In ECHAM5, the 


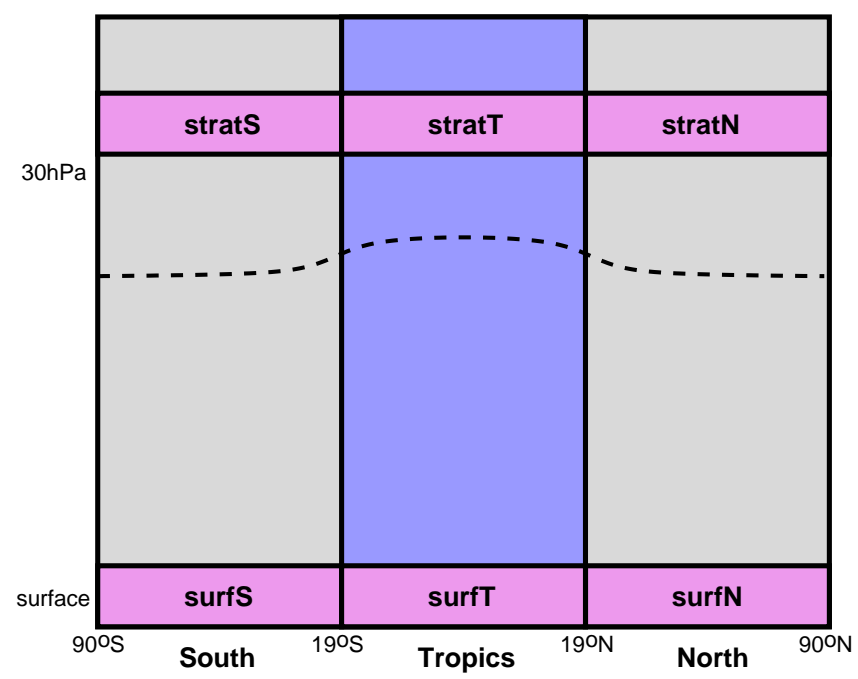

Fig. 1. Schematic diagram showing the independent idealized tracer source regions. The grey shaded areas are the "north" (N) and "south" (S) regions. The blue shaded region is the "tropics" (T). The surface tracers (surfN, surfT and surfS) are introduced at the lowest model level, while the stratosphere tracers (stratN, stratT and stratS) are emitted at $30 \mathrm{hPa}$ level. The broken line represent the tropopause. Note that the diagram is not drawn to scale.

characteristic time scale of this relaxation is set to 3 hours and does not change during the course of the simulation. The atmospheric forcing (surface pressure, temperature, vorticity, and divergence) is then obtained from numerical weather prediction models with data assimilation, e.g. the ECMWF 40years re-analysis data (ERA40, Simmons and Gibson, 2000).

\subsection{Experiment description}

We consider six independent idealized tracers, each constrained to have constant mass mixing ratio of 1 in its respective source region (see Fig. 1). Each of the tracers decays with a fixed globally uniform lifetime everywhere. At each time step, the tracer concentration is restored to 1 in the source region, whenever the tracer is depleted, either due to decay or transport out of the source region. This ensures that the fraction of the tracer that is transported out of the source region is immediately replaced. This is equivalent to prescribing a source which is proportional to the temporally varying net outflow from the region. Our method is not suitable to diagnose redistribution of tracers within the source region and recirculation back into the source region).

Horizontally, we divide the earth surface into three equalarea latitude bands called "north" $(\mathrm{N})$, "tropics" (T) and "south" (S), following Bowman and Carrie (2001); Bowman and Erukhimova (2004). "North" refers to the region north of $19^{\circ} \mathrm{N}$, the region south of $19^{\circ} \mathrm{S}$ is "south" and the region in the latitude bands in-between $19^{\circ} \mathrm{N}$ and $19^{\circ} \mathrm{S}$ is "tropics". Vertically we inject the tracers at two different altitude regimes (which we termed: "surface" and "stratosphere"). The "surface" tracers have their source at the lowest model level, while the "stratosphere" tracers were defined at model levels that corresponds to $30 \mathrm{hPa}$, which is the second level in the vertical resolutions L19 and L31 used in the simulations.

As ECHAM5 uses a hybrid sigma-pressure coordinate system, the actual pressure or altitude of the tracer release varies. At each time step, the atmospheric pressure, $P$ of the layer mid-point, $z$ in a given location and time, $t$ is given by: $P(x, y, z, t)=A(z)+B(z) P_{s}(x, y, t)$; where $P_{s}$ is the surface pressure, $x$ is the longitude, and $y$ is the latitude. The vertical coordinate parameters $A(z)$ and $B(z)$ of the L19 and L31 are given in Table A1. L31 has the same hybrid coefficients $A$ and $B$ for the two topmost and lowermost levels, but finer resolution in the free troposphere. We note that the vertical extent of the two levels we chose to release the idealized tracers is the same in both vertical resolutions.

Henceforth, we will abbreviate the tracer names by combining their vertical and horizontal source region names; for example surfT is the surface tracer with source region in the tropics, while stratN stands for the tracer which is kept at constant concentration in the stratosphere Northern Hemisphere (see Fig. 1). All tracers decay with a fixed globally uniform lifetime.

The experiments in this study are performed using a setup similar to the Atmospheric Model Intercomparison Project 2 (AMIP2, Gates et al., 1999) with prescribed sea surface temperatures and sea ice climatologies of the 1990s. Experiments to test the resolution dependency of tracer transport were performed in the resolutions T21L19, T42L19, T42L31, T63L31, and T106L31. All model resolutions were run with a tracer lifetime of 5 months until a dynamical equilibrium was reached and then continued for four years. Additional sensitivity experiments were performed to test the influence of ERA40 meteorology (run T63L31-era40, using a tracer lifetime of 5 months) and to demonstrate the influence of the tracer lifetimes. The impact of tracer lifetime was tested in T63L31 resolution, using tracers with lifetimes of 0.5 and 50 months, respectively. For the tracers with lifetimes of 5 and 0.5 months one year was sufficient spin-up time. The 50-months-lifetime tracer needed a spin-up of 9 years in order to reach a steady state in the stratosphere. Therefore, the experiments involving tracers with 50 months lifetime was run for a total of 13 years.

\section{The influence of model configuration on the global transport characteristics}

The objective of this section is to characterise the sensitivity of the export flux of a tracer from its source region $i$ into the global atmosphere. Across all the resolutions, the tracer lifetime $\tau$ is set as 5 months, which roughly corresponds to the lifetime of $\mathrm{CO}$ in the troposphere. 

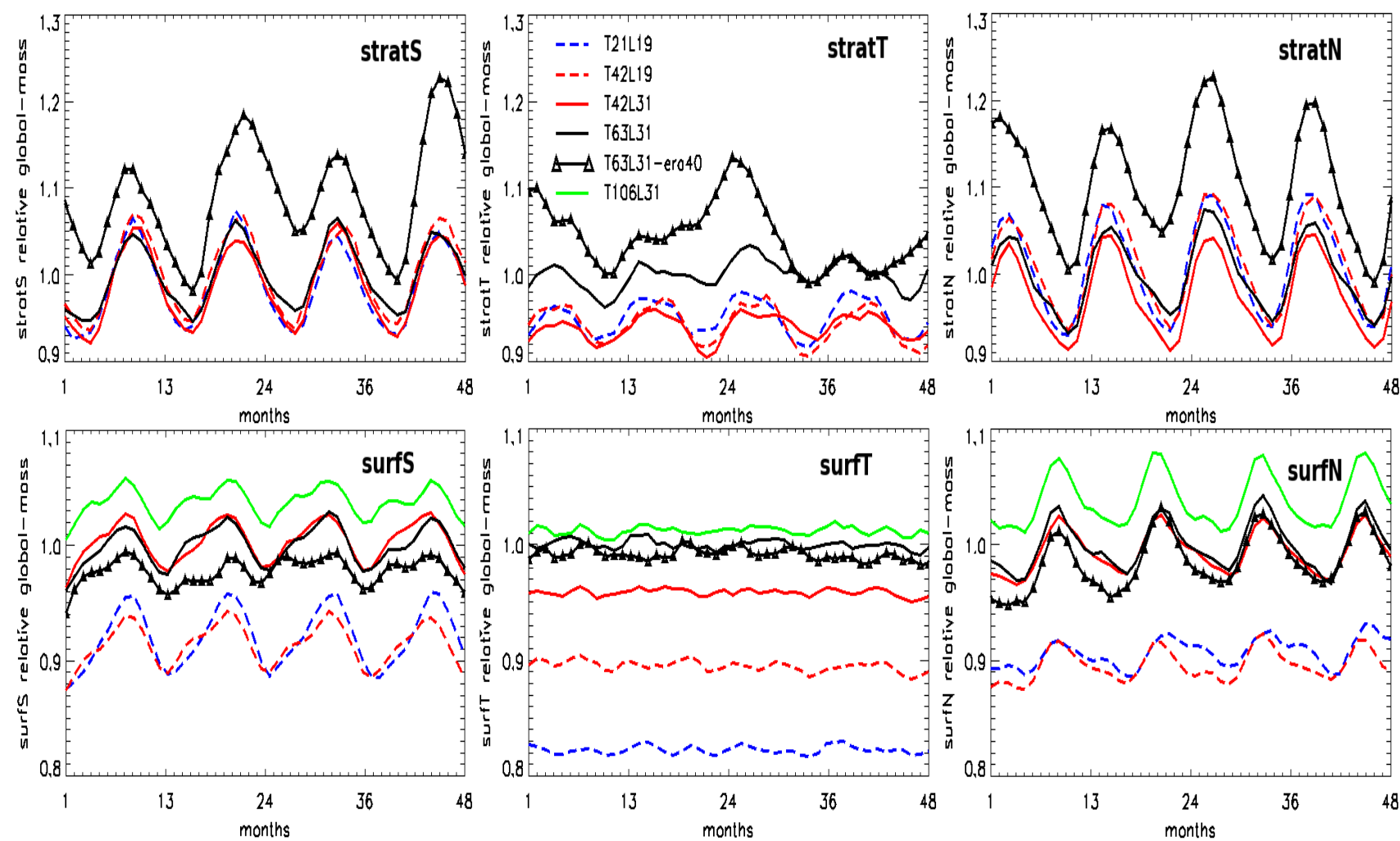

Fig. 2. The resolution comparison index, $R_{i, r}=m_{i, r} / \hat{m}_{i, \mathrm{~T} 63 \mathrm{~L} 31}$ for $r=\mathrm{T} 21 \mathrm{~L} 19$, T42L19, T42L31, T63L31, T63L31-era40 and T106L31. Note that $R$ compares the source region adjusted global-mass of a tracer to the source region adjusted global mass of the same tracer in the T63L31 resolution. See the text for more details. Note that there are no stratosphere tracers included in the T106L31 resolution due to computational cost. Each line represents the last four years of the respective model run.

According to our formulation (see Sect. 2.2), the tracer mixing ratio is restored to 1 at each time step following the depletion of tracer in the source region, either due to transport out of the source region, or due to chemical decay in the source region. This implies that the global amount of the tracer is influenced by the source region extent, the lifetime of the tracer and the net rate of transport out of the source region. Therefore, for any given tracer with source in region $i$ and resolution $r$, the rate of change of the global mass $M_{i, r}$ is given by:

$\dot{M}_{i, r}(t)=S_{i, r}(t)-\frac{M_{i, r}(t)}{\tau}$

where $S_{i, r}$ is the time-dependent net mass flux out of the source region $i$ in the resolution $r$.

According to our simulation setup, at $t=0, M_{i, r}(0)$ equals the mass of the tracer in the source region. This implies that $M_{i, r}(0)$ is proportional to the source region volume, because the tracer is uniformly distributed in the source region. The volume of the source region depends on the exact location of the grid box boundaries which demarcate the source region in each model resolution. Consequently, for each model resolution $r$, we normalise the quantities in Eq. (1) by di- viding them with $M_{i, r}(0)$. Defining $m_{i, r}=M_{i, r} / M_{i, r}(0)$ and $s_{i, r}=S_{i, r} / M_{i, r}(0)$, we get:

$\dot{m}_{i, r}(t)=s_{i, r}(t)-\frac{m_{i, r}(t)}{\tau}$

We further divide $m_{i, r}$ by $\hat{m}_{i, T 63 L 31}$, which is the 4-year average of $m_{i, T 63 L 31}$, to derive a resolution comparison index, $R_{i, r}$ :

$R_{i, r}(t)=\frac{m_{i, r}(t)}{\hat{m}_{i, T 63 L 31}}$

Figure 2 displays the monthly mean values of this resolution comparison index $R_{i, r}$ for each of the idealized tracers in all model configurations. In order to derive an estimate of the strength of the export fluxes in the respective model resolutions, we apply a quasi-steady state approximation to Eq. (2). We define quasi steady state as the state where there is no significant change in the 4-year mean global-mass of the tracers. We note that surfN and stratS tracers appear not to reach the quasi steady state after the end of the simulation period, but this error is negligible in this context. Using this assumption, the integration of the left hand side of Eq. (2) over the last 4 
years of the simulations simply goes to zero, and this results in:

$\bar{s}_{i, r}=\frac{\bar{m}_{i, r}}{\tau}$

where the bar indicates the 4-year average. Substituting Eq. (4) into Eq. (3) yields $\bar{R}_{i, r}=\left(k \bar{s}_{i, r}\right)$, where $k=\tau / \hat{m}_{i, T 63 L 31}$ is a constant. This derivation shows that the average values of $R_{i, r}$ in Fig. 2 directly corresponds to the strength of the export fluxes in the respective model resolution.

The global transport of the surface tracers from their source regions exhibits both horizontal and vertical resolution dependency, especially at the tropical region, where there exists a systematic increase in tracer transport from T21L19 through T106L31. However, there is no clear distinction between the amount of surfN and surfS tracers transported in the T21L19 and T42L19, or T42L31 and T63L31, which means that transport from the surface northern and southern latitudes is much more influenced by the amount of vertical levels of the model.

The stratospheric tracers (stratN, strat $\mathrm{T}$, and strat $\mathrm{S}$ ) on the other hand, show no significant difference between the two numbers of vertical levels. There appears to be little influence of the horizontal resolution on global characteristics of the transport of tracers from the stratosphere, except at the tropical stratosphere (stratT), where T63L31 resolution shows between 5-10\% more export that the remaining resolutions. This seemingly lack of resolution dependency of the global characteristics of stratospheric tracers may be due to limited number of vertical levels in the stratosphere in the version of the model used in this study (which only contains 4-5 levels in the stratosphere).

Some of the tracers (i.e. stratS, stratT, stratN, surfS, and surfN) show a distinct seasonal variation in all model resolutions with one distinct minimum and maximum within any certain year. However, surfT tracer exhibits a semi-annual cycle (with two minimums and maximums within any given year) of lesser amplitude than those observed in the surfN and surfS tracers. The semi-annual cycle is closely connected to the strong forcing exerted by the passing of the sun across the equator twice every year. The seasonal cycle of the southern tracers (surfS, stratS) exhibit slight changes when the lifetime is reduced to 0.5 month or increased to 50 months (not shown), but the changes are more pronounced in the tropical and northern tracers (surfT, surfN, stratT, and stratN). This is consistent with the relation between tracer lifetime and their concentration variability (e.g. see Derwent et al., 1998), short-lived tracers has larger seasonal variability than those of the longer-lived tracers.

Globally, the use of the ERA40 data leads to a lower transport out of the source region at the surface, where the tracers exhibit a relatively small differences of between 0 to $-3 \%$ compared to the AMIP2-style T63L31 simulation. The relaxation of the atmospheric dynamics of the model to ERA40 data (i.e. run T63L31-era40) yields values of $R_{i, r}$ which are about $10 \%$ higher for the stratosphere tracers. In addition, the T63L31-era40 run exhibits the Quasi Biennial Oscillation (QBO) in the strat T tracer. The presence of a QBO in this simulation is a consequence of the assimilation procedure used to generate the ERA40 data. The versions of the ECHAM5 model used for our experiments do not resolve the stratosphere (due to very few layers in the stratosphere) and hence cannot simulate the QBO, which could only be generated in the middle atmosphere ECHAM5 (MAECHAM5) model. The use of ERA40 data also led to a higher interannual variability of the stratosphere tracers, where $R$ varies by no more than $12 \%$ from year to year in the climate run and up to $24 \%$ in the ERA40 run.

The global characteristic of transport evaluated in this section shows little difference between the T42L31 and the T63L31 resolutions, while the difference between the T42L19 and T42L31 resolutions can be up to $10 \%$, especially in the troposphere. This suggests that it is appropriate to use the T42L31 resolution for computationally expensive tropospheric chemistry simulations with ECHAM5 (e.g. Aghedo et al., 2007). We note, however, that Roeckner et al. (2006) find that the simulated mean climate state diverges when the number of vertical levels is increased in the T42 horizontal resolution, whereas it converges to a more realistic climate state in the more expensive T63L31 resolution.

\section{Source-receptor relationships}

\subsection{Meridional transport}

Table 1 lists the fraction of tracer mass exported from source region $\mathrm{i}$ into the atmospheric column of the receptor regions $\mathrm{S}, \mathrm{T}$, and $\mathrm{N}$ after the 4 years of the simulation.

For the three surface tracers, about half of the total tracer mass remains in the zonal band where it originated from, and there is no systematic dependence on the model resolution (except surfT tracer, which decreases as model resolution increases). The meridional transport of surface tracers is stronger in the models with finer resolution, as can be seen from the larger fractions of the surfN and surfS tracers in the $\mathrm{S}$ and $\mathrm{N}$ regions, respectively. As a consequence, the fractions of surfN and surfS tracers in the tropical band decrease in the models with finer resolution. This is consistent with enhanced advection out of the tropics (surfT to $\mathrm{N}$ and surfT to $S$ ) in the models with finer resolution. The T106L31 resolution does not follow this general tendency, and could be related to differences in the physical parameters of the model for the individual resolutions. In this case, the changes beyond T42L31 should be considered insignificant.

In the stratosphere, about $75-84 \%$ of the stratN and stratS tracers remain in the source region, while for the strat $\mathrm{T}$ tracer this fraction is only about 50\%. Meridional transport at $30 \mathrm{hPa}$ is much slower than at the surface (for example, only 
Table 1. The amount of tracers found in the three regions - N, T and S, for all simulations (in \%) during the last 4-year of the simulations. Note that there are no stratosphere tracers included in the T106L31 resolution due to computational cost. The values in bold face show an anomaly in the increase/decrease trend.

\begin{tabular}{|c|c|c|c|c|c|c|c|c|}
\hline \multirow[b]{2}{*}{ Tracers and receptor region } & \multirow[b]{2}{*}{ T21L19 } & \multicolumn{3}{|c|}{ Resolutions (5 months lifetime) } & \multirow[b]{2}{*}{ T106L31 } & \multirow{2}{*}{$\begin{array}{c}\text { Meteorology (5 months lifetime) } \\
\text { T63L31-era40 }\end{array}$} & \multicolumn{2}{|c|}{ Lifetime (T63L31) } \\
\hline & & T42L19 & T42L31 & T63L31 & & & 0.5 month & 50 months \\
\hline \multicolumn{9}{|l|}{ Surface tracers } \\
\hline surfN to $S$ & 12.3 & 13.5 & 15.7 & 16.3 & 15.9 & 15.2 & 1.2 & 30.8 \\
\hline surfS to $\mathrm{N}$ & 12.7 & 13.9 & 15.9 & 16.6 & 16.0 & 15.5 & 1.5 & 30.7 \\
\hline surfN to $T$ & 34.9 & 31.2 & 31.8 & 30.8 & 31.3 & 30.7 & 18.3 & 32.6 \\
\hline surfS to $T$ & 34.9 & 32.3 & 32.6 & 31.4 & 32.3 & 32.0 & 19.2 & 32.8 \\
\hline surfT to $\mathrm{N}$ & 25.6 & 26.8 & 27.6 & 28.4 & 27.8 & 27.8 & 14.8 & 32.9 \\
\hline surfT to $S$ & 26.4 & 28.0 & 28.5 & 29.1 & 29.0 & 28.5 & 15.2 & 33.2 \\
\hline \multicolumn{9}{|l|}{ Stratosphere tracers } \\
\hline stratN to $\mathrm{S}$ & 2.8 & 2.2 & 2.3 & 2.3 & - & 5.9 & 0.01 & 22.3 \\
\hline stratS to $\mathrm{N}$ & 3.2 & 2.8 & 2.4 & 1.9 & - & 3.8 & 0.01 & 21.5 \\
\hline stratN to $\mathrm{T}$ & 20.8 & 16.1 & 16.4 & 15.1 & - & 15.5 & 6.7 & 26.4 \\
\hline stratS to $\mathrm{T}$ & 21.3 & 17.1 & 16.7 & 14.5 & - & 14.2 & 6.8 & 25.9 \\
\hline strat $\mathrm{T}$ to $\mathrm{N}$ & 26.5 & 25.9 & 24.0 & 24.6 & - & 28.3 & 8.2 & 33.9 \\
\hline strat $T$ to $S$ & 24.9 & 25.0 & 26.1 & 27.0 & - & 29.5 & 8.8 & 35.5 \\
\hline \multicolumn{9}{|c|}{ Tracers remaining in the source region } \\
\hline surfN in $\mathrm{N}$ & 52.8 & 55.3 & 52.5 & 52.9 & 52.8 & 54.1 & 80.5 & 36.6 \\
\hline surfS in $S$ & 52.4 & 53.8 & 51.5 & 52.0 & 51.7 & 52.5 & 79.3 & 36.5 \\
\hline surf $\mathrm{T}$ in $\mathrm{T}$ & 48.0 & 45.2 & 43.9 & 42.5 & 43.2 & 43.7 & 70.0 & 33.9 \\
\hline stratN in $\mathrm{N}$ & 76.4 & 81.7 & 81.3 & 82.6 & - & 78.6 & 93.3 & 51.3 \\
\hline strat $S$ in $S$ & 75.5 & 80.1 & 80.9 & 83.6 & - & 82.0 & 93.2 & 52.6 \\
\hline strat $\mathrm{T}$ in $\mathrm{T}$ & 48.6 & 49.1 & 49.9 & 48.4 & - & 42.2 & 83.0 & 30.6 \\
\hline
\end{tabular}

about $2-3 \%$ of the stratN and stratS tracers reach the opposite $\mathrm{S}$ and $\mathrm{N}$ sectors, respectively). Model versions with coarse resolution have larger inter-hemispheric transport in the stratosphere (see Table 1), with the exception of strat $\mathrm{T}$ tracer transported into $\mathrm{S}$ region. Interestingly, there is tendency for reduced transport from $\mathrm{N}$ to $\mathrm{S}$ across the resolutions (compare stratN to $\mathrm{S}$ to strat $\mathrm{S}$ to $\mathrm{N}$ in Table 1), with the exception of T63L31 resolution.

Constraining the model with ERA40 data generally leads to a small change of about $1-3 \%$ in the inter-hemispheric exchange of surface tracers. In contrast to its influence at the surface, ERA40 data increases the inter-hemispheric transport in the stratosphere; this increase is about $9-15 \%$ for transport from the tropical region to both hemispheres and about $100 \%$ for long-range exchange between the $\mathrm{N}$ and $\mathrm{S}$ regions. The QBO generated in the T63L31-era40 simulation may have contributed to this high inter-hemispheric mass exchange observed in the stratosphere tracers.

Table 1 also shows that the long-range meridional exchange between the northern and the Southern Hemisphere, and the meridional transport of stratosphere tracers are most sensitive to the tracer lifetime. The largest differences occur between the tracers of 15 days and 5 months lifetime. The 50-month lifetime surface and tropopause tracers are well mixed, therefore the distribution within the regions varies by less than $7 \%$, whereas this variation in the stratosphere tracers is up to $30 \%$.

\subsection{Vertical transport}

The cross tropopause transport of trace species plays a role for the budgets of various trace gases like ozone and halocarbons. As a proxy for cross tropopause transport, we consider the transport of the surface tracers to the stratosphere (i.e. the percentage of surface tracers found above the $50 \mathrm{hPa}$ level) and transport of the stratosphere tracers to below $750 \mathrm{hPa}$ (Table 2). The vertical transport of the surface tracers shows a dependence on the number of vertical levels. The models with fewer vertical levels show larger vertical transport of the surface tracers to the stratosphere. The percentage amount of tropical surface tracer transported to the stratosphere is slightly higher than that transported from its corresponding $\mathrm{N}$ and $\mathrm{S}$ region, which may be due to the influence of convection in the tropics. Generally, a slightly higher vertical exchange is observed in the $\mathrm{NH}$ compared to the $\mathrm{SH}$ most likely due to orographic effects.

The transport of the stratosphere tracers to below $750 \mathrm{hPa}$, however, shows a dependence on both vertical and horizontal resolution. For L19 and L31 models, an increase in 
Table 2. Fraction (in\%, with respect to global tracer mass) of tracer mass exported from various source regions into the atmosphere above $50 \mathrm{hPa}$ and below $750 \mathrm{hPa}$. Note that there are no stratosphere tracers included in the T106L31 resolution due to computational cost. The values in bold face show an anomaly in the increase/decrease trend.

\begin{tabular}{|c|c|c|c|c|c|c|c|c|}
\hline & \multicolumn{5}{|c|}{ Resolutions (5 months lifetime) } & \multirow{2}{*}{$\begin{array}{c}\text { Meteorology (5 months lifetime) } \\
\text { T63L31-era40 }\end{array}$} & \multicolumn{2}{|c|}{ Lifetime (T63L31) } \\
\hline & T21L19 & T42L19 & T42L31 & T63L31 & T106L31 & & 15days & 50 months \\
\hline \multicolumn{9}{|c|}{ surface tracers above $50 \mathrm{hPa}$} \\
\hline surfN & 0.54 & 0.63 & 0.39 & 0.38 & 0.37 & 0.92 & 0.0057 & 3.68 \\
\hline surfT & 0.65 & 0.72 & 0.47 & 0.43 & 0.39 & 1.14 & 0.0124 & 3.74 \\
\hline surfS & 0.46 & 0.54 & 0.35 & 0.33 & 0.32 & 0.81 & 0.0042 & 3.63 \\
\hline \multicolumn{9}{|c|}{ Stratosphere tracers below $750 \mathrm{hPa}$} \\
\hline stratN & 1.47 & 1.80 & 1.32 & 1.35 & - & 2.04 & 0.0004 & 15.63 \\
\hline stratT & 0.95 & 0.90 & 0.76 & 0.89 & - & 1.19 & 0.0008 & 14.04 \\
\hline stratS & 1.05 & 1.16 & 1.07 & 1.11 & - & 1.78 & 0.0003 & 15.32 \\
\hline
\end{tabular}

horizontal resolution leads to an increase in the downward tranport to the troposphere, with the exception of L19 stratT tracers, which shows a slight decrease between the T21 and T42. The comparison of T42L19 and T42L31 resolutions shows that increasing the number of vertical levels acts to reduce the vertical tranport of the stratosphere tracers to the troposphere.

Although ERA40 data have little effect on the vertical mixing of the surface tracers within the troposphere, it increases their vertical transport to the stratosphere by about a factor of 2.5 (Table 2). ERA40 data also increase the transport of stratosphere tracers to below $750 \mathrm{hPa}$ by up to $60 \%$. This is consistent with findings of Van Noije et al. (2004), who investigated the sensitivity of stratosphere-totroposphere exchange towards different meteorological forcing conditions in their chemistry transport model.

Owing to the long residence time of air in the stratosphere relative to our chosen lifetimes, the fraction of the mass exchanged between the stratosphere and the troposphere approaches 0 when the lifetime is short $(0.5$ months $)$. The fraction of the surface tracers transported to above $50 \mathrm{hPa}$ and stratosphere tracers transported to below $750 \mathrm{hPa}$ rises respectively to about $0.4 \%$ and $1 \%$ when the lifetime is increased to 5 months, and to $4 \%$ and $15 \%$ when the lifetime increases to 50 months. The seasonal cycle of the fraction of tracers transported across the tropopause is influenced by the lifetime of the tracer (figure not shown).

\section{Inter-hemispheric transport time}

In this section, we calculate the inter-hemispheric transport time between the Northern and Southern Hemispheres. Earlier studies have shown that the three horizontal divisions we used in this study are relatively well mixed internally, and large-scale exchange between the regions are comparatively

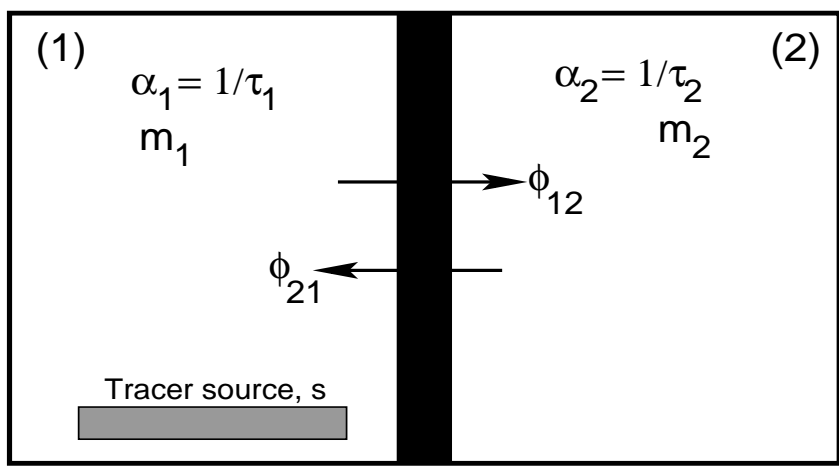

Fig. 3. Conceptual model of tracer transport. See text for details and the description of the parameters.

low. Therefore, we assume that our "tropics" region act as a global-scale mixing barrier between the "north" and the "south" region, such that the values of the surfN and surfS tracers transported to the respective opposite hemisphere can be used to estimate the inter-hemispheric transport time.

We therefore use a conceptual two box-model, with one of the boxes containing the tracer source $s$ as shown in Fig. 3. For each box $i=1,2$, we denote the mass of tracer in the respective box by $m_{i}$. The decay rate of a tracer in box $i$ is $\alpha_{i}=1 / \tau_{i}$, where $\tau_{i}$ is the tracer lifetime. The transport rate of any tracer from box $i$ to box $j, i \neq j$ is $\phi_{i j}=1 / \tau_{i j}$, where $i, j=1,2$. For this general setting, the kinetic equations for the tracer mass are as follows:

$\dot{m}_{1}=-\alpha_{1} m_{1}-\phi_{12} m_{1}+\phi_{21} m_{2}+s$

$\dot{m}_{2}=-\alpha_{2} m_{2}-\phi_{21} m_{2}+\phi_{12} m_{1}$

If a tracer with an infinite lifetime and no source (i.e. $\alpha_{1}=$ $\alpha_{2}=0$, and $s=0$ ) attains a spatially uniform distribution in the steady state, $\phi_{12}=\phi_{21}=\phi$. If the tracer is not in the 

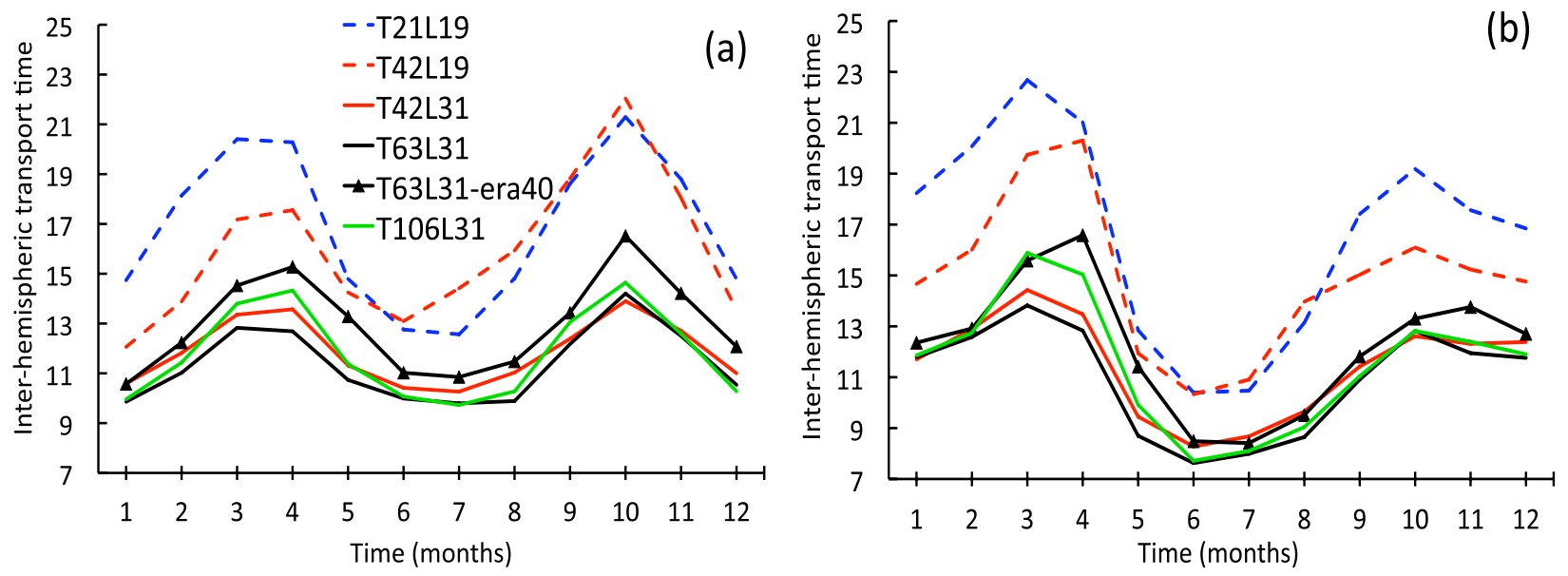

Fig. 4. The 4-year average monthly-mean transport time $\tau$ of the surfN (a) and surfS (b) tracer for different model resolutions.

steady state (which means that either $s \neq 0$ or the tracer has a non-uniform distribution), we can calculate the seasonality of the inter-hemispheric transport time $\tau_{e x}=1 / \phi$ from Eq. (6):

$\tau_{e x}=\frac{m_{1}-m_{2}}{\dot{m}_{2}}$

This is the equation of Prather et al. (1987), which was also used by Kjellström et al. (2000) to determine the interhemispheric transport time from simulated $\mathrm{SF}_{6}$ concentrations.

Figure 4 shows the results of the inter-hemispheric transport time calculated using surfN and surfS tracers in the model resolutions T21L19, T42L19, T42L31, T63L31, T106L31 and the T63L31-era40 version. The minima of the exchange time occur in the months of December to January and June-August. During these months, the exchange of the air masses across the equator is particularly active and the transport times for tracers in both directions are low. This cycle is connected to the position of the ITCZ, which migrates to the north and south of the equator in July-August and January-February respectively. The more rapidly the location of the ITCZ changes, the more intense is the associated exchange of air masses between the large scale northern and southern convective systems.

Figure 4 also shows that the inter-hemispheric exchange time of the models with 19 vertical levels are larger than those with 31 levels by up to 4 months. The figure also shows that the inter-hemispheric exchange time is highly sensitive to the vertical resolution of the model. The use of ECMWF reanalysis data results in a slightly higher interhemispheric transport time of approximately 1 month. The inter-hemispheric transport time calculated from surfN are a couple of days higher than those estimated from the surfS tracers, probably due to the asymmetry of the flow patterns which are strongly influenced by orography.
Table 3. Annual mean value of the inter-hemispheric transport time $\tau_{e x}$ (in months) of the surfN and surfS tracers in various model resolutions.

\begin{tabular}{lcc}
\hline & surfN & surfS \\
\hline T21L19 & 16.8 & 16.7 \\
T42L19 & 15.9 & 14.9 \\
T42L31 & 11.9 & 11.4 \\
T63L31 & 11.4 & 11.0 \\
T63L31-era40 & 13.0 & 12.2 \\
T106L31 & 11.8 & 11.5 \\
\hline
\end{tabular}

The annual mean values of the inter-hemispheric transport time across various model resolutions are given in Table 3. Our inter-hemispheric transport time of $0.9-1.4$ years are similar to the $1.14 \pm 0.16 \mathrm{yr}$ estimated by Czeplak and Junge (1974). These results are lower than the inter-hemispheric exchange time of $1.5-1.7$ years calculated from ${ }^{85} \mathrm{Kr}$ concentrations by Levin and Hesshaimer (1996) with the use of a different two-box model. They explain however, that their result may overestimate the real inter-hemispheric transport time because the interpolation of observation data neglects a decrease in concentration towards higher altitudes and in the stratosphere. The inter-hemispheric transport time in Table 3 and Fig. 4 are considerably longer than the inter-hemispheric exchange time estimated from $\mathrm{SF}_{6}$ tracers in the ECHAM4 model by Kjellström et al. (2000).

An analysis of the cross tropopause transport time is not possible within this study, due to the complex transport patterns in the upper troposphere and lower stratosphere which are not sufficiently captured by our small number of idealized tracers. 


\section{Summary and conclusions}

The influence of model resolution, ERA40 meteorology and the lifetime on the transport of tracers in ECHAM5 has been examined using 6 tracers defined at different horizontal and vertical regions. Transport of the surface tracers is both influenced by the horizontal and vertical resolution of the model, especially at the tropical region, where it monotonically increases with higher number of both resolutions. Whereas, the transport of stratospheric tracers show no such dependence on model resolution, except in the tropical stratosphere, where an increase in the the horizontal resolution to T63 causes between $5-10 \%$ increase in net export of tracers. This may be connected with our models limited number of vertical levels in the stratosphere.

We found a decrease in the mean meridional transport of surface tracers in the coarse resolution models, due to an enhanced vertical mixing, except for the advection into the tropical region, which shows an inconsistent trend between the resolutions. A coarse model resolution leads to enhanced inter-hemispheric transport in the stratosphere, except in the transport from tropical stratosphere to the Southern Hemisphere, which exhibits an increase trend with increasing model resolution.

The use of ERA40 data only slightly affects the interhemispheric transport of surface tracers, however it increases the vertical transport of surface tracers to the stratosphere by up to a factor of 2.5. ERA40 meteorology also increases the long-range inter-hemispheric exchange between the northern and southern stratospheric region by up to $100 \%$, and stratosphere-troposphere transport by up to $60 \%$. ERA40 data led to the generation of Quasi-Biennial Oscillation at the tropical stratosphere, which would not normally be possible in the versions of the ECHAM5 model used in this study. The use of ERA40 data also led to a higher inter-annual variability of the stratosphere tracers, which varies by no more than $12 \%$ from year to year in the climate run and up to $24 \%$ in the ERA40 run.

The long-range inter-hemispheric transport between the northern and the Southern Hemisphere, and the interhemispheric transport of the stratosphere tracers are most affected by the tracers' lifetime. The largest differences are however found between the tracers with lifetimes of 0.5 month and 5 months. The 50-month lifetime surface and tropopause tracers are well mixed, therefore the distribution within the regions varies by less than $7 \%$, and the percentage amount found in our 3-latitudinal regions is between $30-$ $36 \%$ irrespective of their source region. Tracer lifetime also has an influence on the seasonal cycle of the tracers.

The results from the conceptual box model show that it will take between 11 to 17 months for the surface tracer with source in the southern and Northern Hemisphere respectively to be transported to the other hemisphere. These results are consistent with the inter-hemispheric exchange time of $1.14 \pm 0.16 y r$ calculated by Czeplak and Junge (1974). The inter-hemispheric transport is most active in December to January and June to August. The models with the higher vertical resolution in the AMIP2-style runs yield a seasonal cycle of the inter-hemispheric exchange times, which are up to 4 months lower than those of the coarse resolution models, and approximately 1 month lower than a similar model driven with the ECMWF reanalysis data.

We found striking similarities in the performance of the T42 and T63 horizontal resolutions with respect to the transport of tracers, when the model vertical level is increased to 31. This may suggests that despite the lack of added benefits in the zonal mean climate state with respect to T42L19 (Roeckner et al., 2006), the use of T42L31 resolution in the transport of chemical species may present an additional benefit which may be comparable to the more computationally expensive T63L31. Due to the model setup used in this study, additional work that employs tracers with known atmospheric emissions and concentrations is needed in order to provide some recommendations on the "best" ECHAM5 model resolution.

\section{Appendix A}

\section{ECHAM5 hybrid sigma-pressure vertical coordinate}

The vertical coordinate in the ECHAM5 model uses a hybrid sigma-pressure coordinate system. The atmospheric pressure, $P$ at the level mid-point, $z$ in a given location and time, $t$ is given by: $P(x, y, z, t)=A(z)+B(z) P_{s}(x, y, t)$; where $P_{s}$ is the surface pressure, $x$ is the longitude, and $y$ is the latitude. The vertical coordinate parameters $A(z)$ and $B(z)$ of the L19 and L31 are as given in Table A1. 
Table A1. The ECHAM5 model vertical coordinate parameters of the L19 and L31 vertical levels. The entries show the respective layer mid-points.

\begin{tabular}{|c|c|c|c|c|}
\hline \multirow{2}{*}{$\begin{array}{c}\text { Level } \\
\text { mid-point, } z\end{array}$} & \multicolumn{2}{|c|}{ L19 } & \multicolumn{2}{|c|}{ L31 } \\
\hline & $A(z)[\mathrm{Pa}]$ & $B(z)$ & $A(z)[\mathrm{Pa}]$ & $B(z)$ \\
\hline 1 & 1000.0000 & 0.00000000 & 1000.000000000 & 0.00000000000 \\
\hline 2 & 3000.0000 & 0.00000000 & 3000.000000000 & 0.00000000000 \\
\hline 3 & 5023.0555 & 0.00016949665 & 5000.000000000 & 0.00000000000 \\
\hline 4 & 7157.0195 & 0.00184809015 & 7000.000000000 & 0.00000000000 \\
\hline 5 & 9438.7190 & 0.0082136135 & 8988.068359375 & 0.000195429078303275 \\
\hline 6 & 11730.3050 & 0.023573595 & 10898.337890625 & 0.00165527942590415 \\
\hline 7 & 13774.8000 & 0.05236349 & 12625.966796875 & 0.0060569163179025 \\
\hline 8 & 15279.8150 & 0.098283265 & 14083.8750000 & 0.0147566441446543 \\
\hline 9 & 15988.6850 & 0.16355605 & 15212.78125 & 0.0286470074206591 \\
\hline 10 & 15736.5800 & 0.2483575 & 15977.908203125 & 0.0482312496751547 \\
\hline 11 & 14489.1900 & 0.3504644 & 16365.806640625 & 0.0736913084983826 \\
\hline 12 & 12361.5100 & 0.4651707 & 16381.3125000 & 0.104949295520782 \\
\hline 13 & 9614.3520 & 0.58552005 & 16044.609375 & 0.141722559928894 \\
\hline 14 & 6626.1430 & 0.70290315 & 15388.43359375 & 0.183572381734848 \\
\hline 15 & 3837.5555 & 0.808068 & 14455.39453125 & 0.229945927858353 \\
\hline 16 & 1666.5820 & 0.89259225 & 13295.40625 & 0.280211985111237 \\
\hline 17 & 391.5975 & 0.95086605 & 11963.26171875 & 0.333690196275711 \\
\hline 18 & 0.0000 & 0.98263335 & 10516.318359375 & 0.38967365026474 \\
\hline 19 & 0.0000 & 0.99614075 & 9012.30859375 & 0.447445213794708 \\
\hline 20 & & & 7507.275390625 & 0.50628736615181 \\
\hline 21 & & & 6053.626953125 & 0.565485417842865 \\
\hline 22 & & & 4698.31591796875 & 0.624324411153793 \\
\hline 23 & & & 3481.1435546875 & 0.682079493999481 \\
\hline 24 & & & 2433.18725585938 & 0.737999826669693 \\
\hline 25 & & & 1575.34753417969 & 0.791286110877991 \\
\hline 26 & & & 917.019409179688 & 0.841061413288116 \\
\hline 27 & & & 454.8876953125 & 0.886335849761963 \\
\hline 28 & & & 171.845024108887 & 0.925964534282684 \\
\hline 29 & & & 36.0317916870117 & 0.958599209785461 \\
\hline 30 & & & 0.000000000 & 0.982633352279663 \\
\hline 31 & & & 0.000000000 & 0.996140748262405 \\
\hline
\end{tabular}

Acknowledgements. This work was carried out during the doctoral work of AMA, sponsored by the ZEIT foundation through the International Max Planck Research School on Earth System Modelling (IMPRS-ESM). SR and MGS acknowledge funding from the EU project RETRO (EVK2-CT-2002-00170). We are grateful to the ECMWF for providing the reanalysis data, and to Erich Roeckner, Marco Giorgetta, and Kevin W Bowman for their useful comments, and also to Luis Kornblueh for the technical assistance. The model runs were performed on the Sun Computing system (YANG) at the Max Planck Institute for Meteorology, Hamburg and the NEC SX-6 computer at the German Climate Computing Centre ("Deutsches Klimarechenzentrum"). We thank the editor, Peter Haynes, and appreciate the comments of Kenneth Bowman, and three other anonymous referees, which have significantly improve the manuscript.

Edited by: P. Haynes

\section{References}

Aghedo, A. M., Schultz, M. G. and S. Rast: The influence of African air pollution on regional and global tropospheric ozone, Atmos. Chem. Phys., 7, 1193-1212, 2007, http://www.atmos-chem-phys.net/7/1193/2007/.

Asselin, R.: Frequency filter for time integrations. Mon. Weather Rev. 100, 487-490, 1972.

Austin, J., Butchart N., and Swinbank, R.: Sensitivity of ozone and temperature to vertical resolution in a GCM with coupled stratospheric chemistry, Q. J. Roy. Meteorol. Soc., 123, 1405-1431, 1997.

Bowman, K. P. and Carrie, G. D.: The mean-meridional transport circulation of the troposphere in an idealized GCM, J. Atmos. Sci., 59, 1502-1514, 2001.

Bowman, K. P. and Erukhimova, T.: Comparison of global-scale Lagrangian transport properties of the NCEP Reanalysis and CCM3, J. Climate, 17, 1135-1145, 2004. 
Czeplak, G. and Junge C.: Studies of interhemispheric exchange in the troposphere by a diffusion model, Adv. Geophys., 18B, 57-72, 1974.

Denning, A. S., Holzer, M., Gurney, K. R., Heimann, M., Law, R. M., and coauthors: Three-dimensional transport and concentration of $\mathrm{SF}_{6}$ : A model intercomparison study (TransCom 2), Tellus 51B, 266-297, 1999.

Derwent, R. G., Simmonds, P. G., Seuring, S., and Dimmer, C.: Observation and interpretation of the seasonal cycles in the surface concentrations of ozone and carbon monoxide at Mace Head, Ireland from 1990 to 1994, Atmos. Environ., 32(2), 145-157, 1998.

Gates W. L., Boyle, J. S., Covey, C., Dease, C. G., Doutriaux, C. M., Drach, R. S., M. Fiorino, M., Gleckler, P. J., J. J. Hnilo, J. J., Marlais, S. M., Phillips, T. J., Potter, G. L., Santer, B. D., Sperber, K. R., Taylor, K. E., and Williams, D. N.: An Overview of the Results of the Atmospheric Model Intercomparison Project (AMIP I). Bulletin of the American Meteorological Society, 80(1), pp. 2955, 1999.

Genthon, C. and Armengaud, A.: Radon 222 as a comparative tracer of transport and mixing in two general circulation models of the atmosphere, J. Geophys. Res., 100(D2), 2849-2866, 1995.

Gray, S. L.: A case study of stratosphere to troposphere transport: The role of convective transport and the sensitivity to model resolution, J. Geophys. Res., 108(D18), 4590, doi:10.1029/2002JD003317, 2003.

Gurney, K. R., Law, R. M., Denning, A. S., Rayner, P. J., Baker, D. and coauthors.: Towards robust regional estimates of $\mathrm{CO} 2$ sources and sinks using atmospheric transport models, Nature 415, 626-630, 2002.

Gurney, K. R., Law, R. M., Denning, A. S., Rayner, P. J., Baker, D. and coauthors: TransCom $3 \mathrm{CO}_{2}$ inversion intercomparison: 1 . Annual mean control results and sensitivity to transport and prior flux information, Tellus 55B, 555-579, 2003.

Hall, T. M., Waugh, D. W., Boering, K. A., and Plumb, R. A.: Evaluation of transport in stratospheric models, J. Geophys. Res., 104(D15), 18815-18839, 1999.

Hoke, J. E. and Anthes, R. A.: The initialization of numerical models by a dynamic-initialization technique Mon. Weather Rev., 104(12), 1551-1556, 1976.

Jacob, D. J., Prather, M. J., Rasch, P. J., Shia, R.-L., Balkanski, Y. J., Beagley, S. R., et al.: Evaluation and intercomparison of global atmospheric transport models using ${ }^{222} \mathrm{Rn}$ and other short-lived tracers. J. Geophys. Res., 102(D5), 5953-5970, doi:10.1029/96JD02955, 1997.

Jeuken, A. B. M., Siegmund, P. C., Heijboer, L. C., Feichter, J., and Bengtson, L.: On the potential of assimilating meteorological analysis in a climate model for the purpose of model validation, J. Geophys. Res., 101, 16939-16950, 1996.

Kjellström, E., Feichter, J., and Hoffman, G.: Transport of $\mathrm{SF}_{6}$ and ${ }^{14} \mathrm{CO}_{2}$ in the atmospheric general circulation model ECHAM4, Tellus 52B, 1-18, 2000.

Lanser D. and Verwer, J.G.: Analysis of operator splitting for advection-diffusion-reaction problems from air pollution modelling, J. Comp. Appl. Math., 111, 201-216, 1999.

Levin, I. and Hesshaimer, V.: Refining of atmospheric transport model entries by the globally observed passive tracer distribution of ${ }^{85}$ Krypton and sulfur hexafluoride $\left(\mathrm{SF}_{6}\right)$, J. Geophys. Res., 101(D11), 16745-16755, 1996.
Lin, S.-J. and Rood, R. B.: Multidimensional Flux-Form SemiLagrangian Scheme. Mon. Wea. Rev., 124, 2046-2070, 1996.

Lott, F., and M. J. Miller: A new subgrid-scale orographic drag parameterization: Its formulation and testing, Q. J. Roy. Meteorol. Soc., 123, 101-127, 1997.

Mesinger, F. and Arakawa, A.: Numerical methods used in atmospheric models. Global Atmospheric Research Programme (GARP) Publication Series 17, World Meteorological Organisation, 1976.

Nordeng, T. E.: Extended versions of the convective parameterization scheme at ECMWF and their impact on the mean and transient activity of the model in the tropics. Technical memorandum No. 206, European Centre for Medium-range Weather Forecasts (ECMWF), Reading, UK, 1994.

Prather, M., McElroy, M., Wofsy, S., Russell, G., and Rind, D.: Chemistry of the global troposphere: Fluorocarbons as tracers of air motion, J. Geophys. Res. 92, 6579-6613, 1987.

Robert, A. J.: A stable numerical integration scheme for the primitive meteorological equations, Atmos. Ocean., 19, 35-46, 1981.

Robert, A. J.: A semi-Lagrangian and semi-implicit numerical integration fscheme for the primitive meteorological equations, J. Meteor. Soc. Jpn., 60, 319-325, 1982.

Robert, A. J., Henderson, J. and Turnbull, C.: An implicit time integration scheme for baroclinic models in the atmosphere, Mon Weather Rev., 100, 329-335, 1972.

Roeckner, E, G. Bäuml, L. Bonaventura, R. Brokopf, M. Esch, M. Giorgetta, S. Hagemann, I. Kirchner, L. Kornblueh, E. Manzini, A. Rhodin, U. Schlese, U. Schulzweida, A. Tompkins (2003): The atmospheric general circulation model ECHAM 5. PART I: Model description, Max-Planck Institute for Meteorology report No 349, Hamburg, Germany, 127 pp., 2003.

Roeckner, E, Brokopf, R., Esch, M., Giorgetta, M., Hagemann, S., Kornblueh, L., Manzini, E., Schlese, U., Schulzweida, U.: Sensitivity of simulated climate to horizontal and vertical resolution in the ECHAM5 atmosphere model, J. Climate, 19, 3771-3791, 2006.

Simmons, A. J., Burridge, D. M., Jarraud, M., Girard, C., Wergen, W.: The ECMWF medium-range prediction models development of the numerical formulations and the impact of increased resolution, Meteorol. Atmos. Phys., 40, 28-60, doi:10.1007/BF01027467, 1989.

Simmons, A. J and Burridge, D. M.: An energy and angularmomentum conserving vertical finite difference scheme and hybrid vertical coordinates, Mon. Weather Rev., 109, 758-766, 1981.

Simmons, A. J. and Gibson, J. K.: ERA-40 Project plan. ERA40 project report series No 1, 63 pp, 2000.

Stevenson, D. S., Dentener, F. J., Schultz, M. G., Ellingsen, K., van Noije, T. P. C., et al.: Multimodel ensemble simulations of present-day and near-future tropospheric ozone, J. Geophys. Res., 111, D08301, doi:10.1029/2005JD006338, 2006.

Tiedtke, M.: A comprehensive mass flux scheme for cumulus parameterization in large-scale models. Mon. Weather Rev., 117, 1779-1800, 1989.

van Noije, T.P.C., Eskes, H. J., van Weele, M., and van Velthoven, P. F. J.: Implications of the enhanced Brewer-Dobson circulation in European Centre for Medium Range Weather Forecasts 
reanalysis ERA-40 for the stratosphere troposphere exchange of ozone in global chemistry transport models, J. Geophys. Res., 109, D19308, doi:10.1029/2004JD004586, 2004.
Wild, O. and Prather, M. J.: Global tropospheric ozone modelling: Quantifying errors due to grid resolution, J. Geophys. Res., 111, D11305, doi:10.1029/2005JD006605, 2006. 